\title{
Bioinformatics Analysis of the Molecular Mechanism of Aging on Fracture Healing
}

\author{
Shu-Jie Zhao, ${ }^{1}$ Fan-Qi Kong, ${ }^{1}$ Jin Fan, ${ }^{1}$ Ying Chen, ${ }^{2}$ Shuai Zhou, \\ Ming-Xin Xue $\mathbb{C}^{3},{ }^{3}$ and Guo-Yong Yin $\mathbb{C}^{1}$ \\ ${ }^{1}$ Department of Orthopedics, The First Affiliated Hospital of Nanjing Medical University, Nanjing, Jiangsu 210029, China \\ ${ }^{2}$ Affiliated Hospital of Nanjing University of TCM, Nanjing, Jiangsu 210029, China \\ ${ }^{3}$ Department of Massage, The First Affiliated Hospital of Nanjing Medical University, Nanjing, Jiangsu 210029, China
}

Correspondence should be addressed to Ming-Xin Xue; jshtcmxmx@sina.com and Guo-Yong Yin; yggsubmission@126.com

Received 26 May 2018; Revised 26 November 2018; Accepted 2 December 2018; Published 16 December 2018

Academic Editor: Momiao Xiong

Copyright (C) 2018 Shu-Jie Zhao et al. This is an open access article distributed under the Creative Commons Attribution License, which permits unrestricted use, distribution, and reproduction in any medium, provided the original work is properly cited.

\begin{abstract}
Increasing age negatively affects different phases of bone fracture healing. The present study aimed to explore underlying mechanisms related to bone fracture repair in the elderly. GSE17825 public transcriptome data from the Gene Expression Omnibus database were used for analysis. First, raw data were normalized and differentially expressed genes (DEGs) were identified. Next, Kyoto Encyclopedia of Genes and Genomes (KEGG) and Gene Ontology (GO) analyses were implemented to evaluate pathways and DEGs. A protein-protein interaction (PPI) network was then constructed. A total of 726, 861, and 432 DEGs were identified between the young and elderly individuals at 1, 3, and 5 days after fracture, respectively. The results of GO, KEGG, and PPI network analyses suggested that the inflammatory response, Wnt signaling pathway, vascularization-associated processes, and synapticrelated functions of the identified DEGs are markedly enriched, which may account for delayed fracture healing in the elderly. These findings provide valuable clues for investigating the effects of aging on fracture healing but should be validated through further experiments.
\end{abstract}

\section{Introduction}

The elderly population has been steadily growing worldwide, and persons aged $\geqslant 65$ years are projected to comprise $16.9 \%$ of the American population by 2030 [1,2]. Bone fractures are a common orthopedic problem, with a residual lifetime fracture risk in a 60 -year-old person reported to be $29 \%$ in males and $56 \%$ in females, and pose an extensive medical and socioeconomic burden $[3,4]$. The elderly are at a higher risk of delayed healing or non-union after bone fracture, which can have severe and systemic consequences in this age group $[5,6]$. Taking into account this reality, a better understanding of age-related effects on fracture healing is critical for the development and optimization of effective therapeutic treatments.

Fracture healing is a complex but well-orchestrated process that can be temporally divided into three partially overlapping processes, namely, inflammation, repair, and remodeling [7-9]. In the initial phase, temporal control of the inflammatory response is necessary to initiate healing [10]. The inflammatory phase involves stimulating angiogenesis, attracting mesenchymal stem cells (MSCs), and promoting their differentiation, as well as enhancing extracellular matrix synthesis [11].

However, changes in the inflammatory system occur with age. In older animal models, prolonged inflammation results in delayed chondrogenesis and smaller callus size $[12,13]$. Age-related decline of MSC quantity and quality may negatively affect the ability of these cells to support fracture healing, as demonstrated in humans and animal models $[14,15]$. Delayed fracture healing is also associated with reduced growth factor levels and impaired angiogenesis, which are also characteristic of advanced age [16-18]. Such age-related changes are accompanied by changes in the regulation of critical molecular events involved in fracture healing, particularly those occurring early in the bone repair 
process $[19,20]$. However, molecular changes that occur in a temporal manner are yet to be elucidated.

In this study, transcriptional analysis of GSE17825 was used to illustrate molecular mechanisms by which age affects fracture healing. Specifically, differentially expressed genes (DEGs) at three different time-points, namely, 1, 3, and 5 days after fracture, were evaluated by pathway and functional enrichment analysis. A protein-protein interaction (PPI) network was then constructed using these DEGs. These analyses revealed several molecular mechanisms that may contribute to age-related changes in fracture healing.

\section{Materials and Methods}

2.1. Transcriptome Data. Transcriptome profiles of GSE17825, which was submitted by Liang et al., were obtained from the National Centre of Biotechnology Information (NCBI) Gene Expression Omnibus database (GEO, http://www.ncbi.nlm.nih.gov/geo/). GSE17825, which comprises a total of 18 chips, including nine old group (8-month-old mice) and nine young group (6-week-old mice) samples, was based on the platform of the PL1261 Affymetrix Mouse Genome 4302.0 Array. For each group, fracture calluses were dissected and total RNA was isolated at each of the three time-points ( $n=3$ per time-point).

2.2. Data Preprocessing. The robust multichip average algorithm from the Oligo package (version 1.42.0, http://www .bioconductor.org) was used to preprocess non-normalized raw data by background correction normalization, probe summary, and $\log 2$ transformation [21]. Probe identification numbers (IDs) were then converted into gene symbols, and if different probes were annotated to the same gene, then the average value served as the gene's expression level.

2.3. Identification and Analysis of DEGs. In this study, several paired groups were compared as follows: (i) old vs. young group at 1 day; (ii) old vs. young group at 3 days; and (iii) old vs. young group at 5 days. Student's t-test was used for comparisons. Genes with fold changes $>2.0$ and $\mathrm{P}<0.05$ were considered DEGs.

2.4. Pathway and Functional Enrichment Analysis. The Database for Annotation, Visualization, and Integrated Discovery (DAVID 6.8; http://david.abcc.ncifcrf.gov) can provide functional annotation of enormous quantities of genes derived from different genomic resources. In this study, DAVID was used to conduct Kyoto Encyclopedia of Genes and Genomes (KEGG) pathway and Gene Ontology (GO) analyses to determine significant DEGs [22]. GO terms were determined as categories of biological processes (BPs), and $\mathrm{P}$ $<0.05$ was defined as the cut-off criterion.

2.5. PPI Network Construction. The Search Tool for the Retrieval of Interacting Genes/Proteins (STRING) database (http://www.string-db.org) was used to evaluate potential protein-protein interactions. Cytoscape is an open-source tool for network visualization of genes, proteins, and other types of BPs [23]. The default confidence cut-off of 400 was used, as previously described [24]. Experimentally validated interactions, as revealed by solid lines, were included, and single nodes without interactions were excluded [24].

\section{Results}

3.1. Data Preprocessing and DEG Screening. Box plots of GSE17825 value distribution before and after data normalization were generated (Figure 1(a)). Data from each sample were generally normalized and cross-comparable following normalization (Figure 1(a)). DEGs between the old and young groups at the three time-points were then analyzed. A total of 618, 162, and 208 DEGs were upregulated and 108, 699, and 224 DEGs were downregulated at 1,3, and 5 days after fracture, respectively (Figure 1(b)); volcano plots were used to visualize the identified DEGs (Figure 1(b)). Finally, heat maps of gene expression values were constructed with color patterns indicating the variability in gene expression between the old and young groups after fracture (Figure 2).

3.2. KEGG Pathway and GO Enrichment Analysis of DEGs. The five most enriched KEGG pathways of up- and downregulated DEGs at each time-point $(1,3$, and 5 days $)$ are presented in Figures 3(a) and 3(b), respectively. At 1 day, the upregulated DEGs were enriched in pathways including neuroactive ligand-receptor interaction $\left(\mathrm{P}=4.65 \times 10^{-4}\right)$, taurine and hypotaurine metabolism $\left(\mathrm{P}=5.02 \times 10^{-3}\right)$, fat digestion and absorption $\left(\mathrm{P}=7.08 \times 10^{-3}\right)$, glycerolipid metabolism $(\mathrm{P}=$ $\left.2.13 \times 10^{-2}\right)$, and dorsoventral axis formation $\left(\mathrm{P}=2.69 \times 10^{-2}\right)$. At 3 days, the upregulated DEGs were primarily involved in ECM-receptor interaction $\left(\mathrm{P}=4.38 \times 10^{-5}\right)$, focal adhesion $\left(\mathrm{P}=3.76 \times 10^{-4}\right)$, regulation of actin cytoskeleton $(\mathrm{P}=5.50$ $\left.\times 10^{-4}\right)$, PI3K-Akt signaling pathway $\left(\mathrm{P}=1.23 \times 10^{-3}\right)$, and phagosome $\left(\mathrm{P}=2.13 \times 10^{-3}\right)$. Finally, at 5 days after fracture, these were involved in complement and coagulation cascades $\left(\mathrm{P}=4.25 \times 10^{-3}\right)$, neuroactive ligand-receptor interaction $\left(\mathrm{P}=2.19 \times 10^{-2}\right)$, cell adhesion molecules $(\mathrm{P}=2.86 \times$ $\left.10^{-2}\right)$, rheumatoid arthritis $\left(\mathrm{P}=4.11 \times 10^{-2}\right)$, and leukocyte transendothelial migration $\left(\mathrm{P}=7.69 \times 10^{-2}\right)$.

At 1 day, the downregulated DEGs were involved in protein digestion and absorption $\left(\mathrm{P}=3.28 \times 10^{-8}\right)$, ECM-receptor interaction $\left(\mathrm{P}=1.33 \times 10^{-5}\right)$, focal adhesion $(\mathrm{P}$ $\left.=9.59 \times 10^{-5}\right)$, platelet activation $\left(\mathrm{P}=1.13 \times 10^{-3}\right)$, and PI3KAkt signaling pathway $\left(\mathrm{P}=1.76 \times 10^{-3}\right)$. At 3 days, these were primarily enriched in pathways related to $\mathrm{ABC}$ transporters $\left(\mathrm{P}=1.12 \times 10^{-2}\right)$, morphine addiction $\left(\mathrm{P}=1.45 \times 10^{-2}\right)$, Wnt signaling pathway $\left(\mathrm{P}=1.61 \times 10^{-2}\right)$, phototransduction $\left(\mathrm{P}=2.82 \times 10^{-2}\right)$, and glycosphingolipid biosynthesis: lacto and neolacto series $\left(\mathrm{P}=2.82 \times 10^{-2}\right)$. Finally, at 5 days after fracture, these were enriched in vascularization-associated pathways including platelet activation $\left(\mathrm{P}=1.43 \times 10^{-5}\right)$, hematopoietic cell lineage $\left(\mathrm{P}=3.86 \times 10^{-5}\right)$, and VEGF signaling pathway $\left(\mathrm{P}=2.89 \times 10^{-2}\right)$.

The 10 most significantly enriched GO terms (BPs) for the identified DEGs are listed in Table 1. At 1 day after fracture, DEGs were involved in development-associated BPs, such as skeletal system development $\left(\mathrm{P}=3.32 \times 10^{-6}\right)$, tissue development $\left(\mathrm{P}=5.61 \times 10^{-6}\right)$, negative regulation of multicellular 


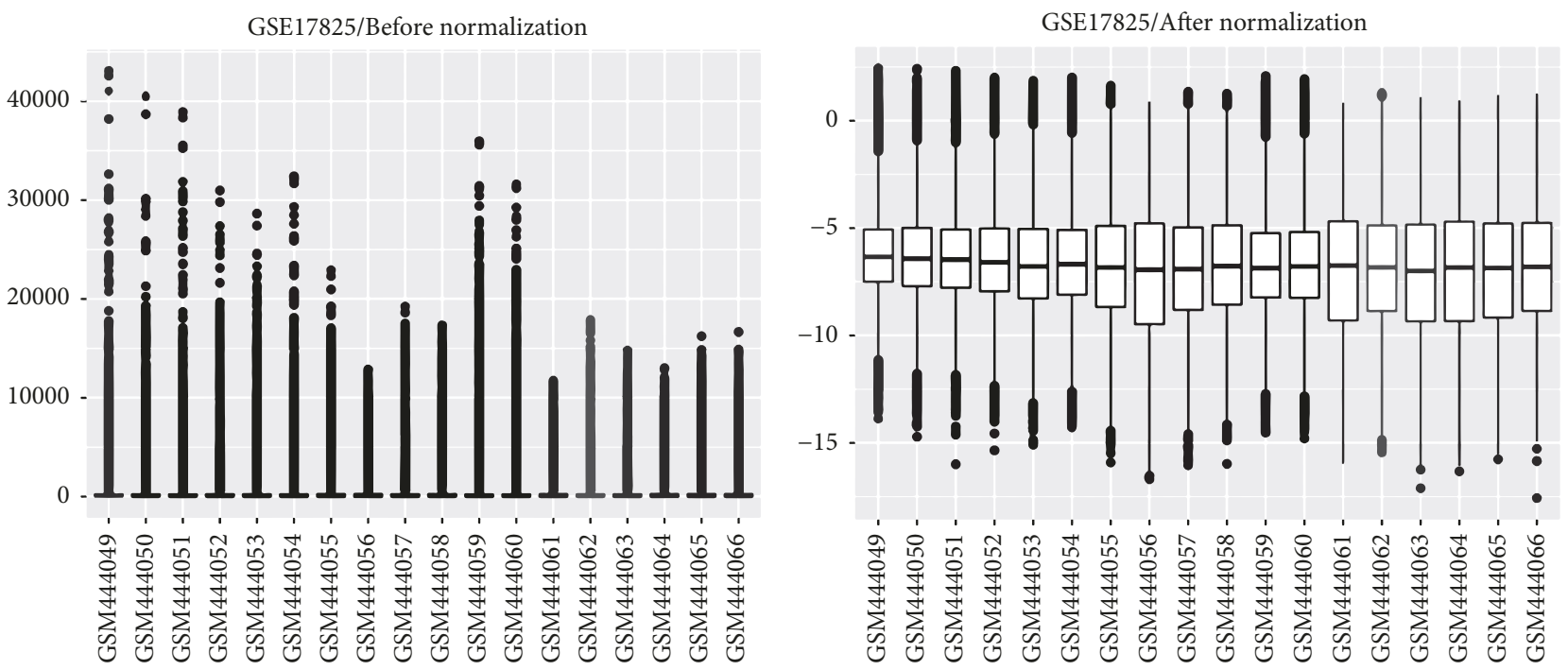

(a)
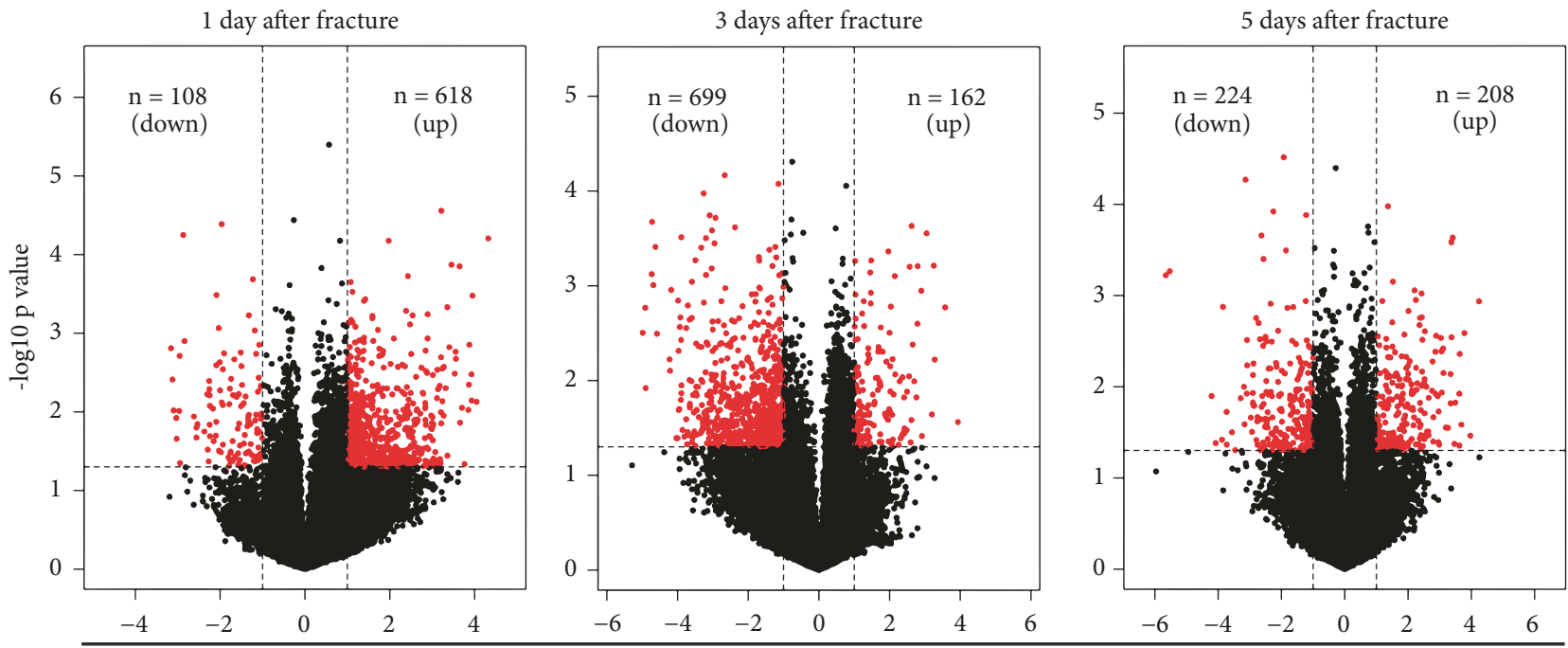

Log2 Fold change

(b)

FiguRE 1: (a) Data normalization of differentially expressed genes (DEGs). Box plots of gene expression in the old and young groups at the fracture site (left panel) before and (right panel) after normalization. (b) Volcano plots at 1, 3, and 5 days of the old vs. young groups.

organismal process $\left(\mathrm{P}=6.69 \times 10^{-6}\right)$, negative regulation of cell development $\left(\mathrm{P}=3.32 \times 10^{-6}\right)$, and trabecular formation $\left(\mathrm{P}=1.04 \times 10^{-4}\right)$. At 3 days after fracture, these were the most enriched in antigen processing and peptide or polysaccharide antigen presentation via MHC class II $\left(\mathrm{P}=5.58 \times 10^{-7}\right)$. At 5 days after fracture, these were predominantly associated with synaptic-related functions, such as anterograde transsynaptic signaling $\left(\mathrm{P}=5.80 \times 10^{-7}\right)$, trans-synaptic signaling $\left(\mathrm{P}=5.98 \times 10^{-7}\right)$, synaptic signaling $\left(\mathrm{P}=7.67 \times 10^{-7}\right)$, neuron-neuron synaptic transmission $\left(\mathrm{P}=1.00 \times 10^{-5}\right)$, and chemical synaptic transmission $\left(\mathrm{P}=4.16 \times 10^{-5}\right)$.

3.3. PPI Network Construction and Functional Module Analysis. PPI network analysis was performed using Cytoscape software. Results revealed that, at 1 day after fracture, DEGs were enriched in platelet-derived growth factors binding, extracellular matrix structural constituent, and the Wnt signaling pathway (Figure 4(a)). At 3 days, DEGs were involved in the Wnt signaling pathway, cell adhesion molecules (CAMs), transmembrane transporter activity, and intrinsic component of membrane (Figure 4(b)). Finally, at 5 days after fracture, DEGs were enriched in complement and coagulation cascades, VEGF signaling pathway, and neuroactive ligand-receptor interaction (Figure 4(c)).

\section{Discussion}

Fracture healing involves multiple biological phases that are characterized by both anabolic and catabolic processes [7]. While aging has a significant impact on skeletal physiology, 


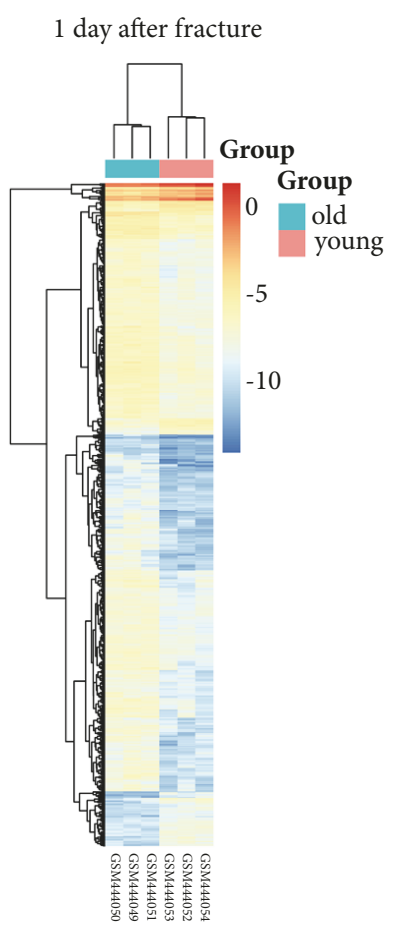

(a)

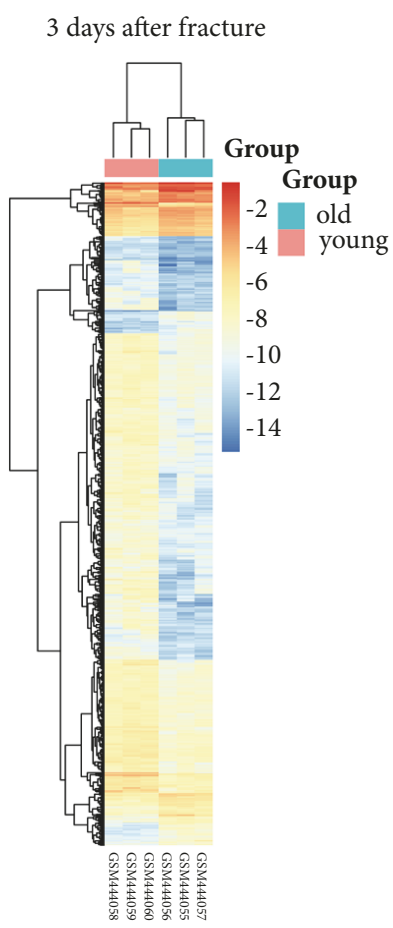

(b)

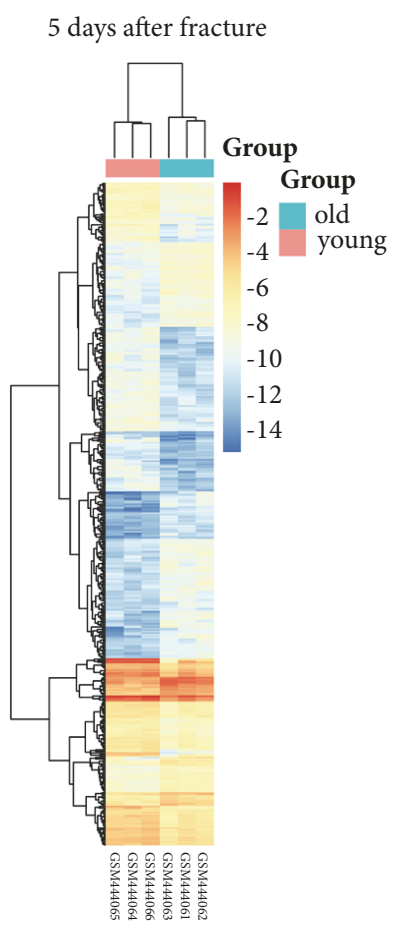

(c)

FIGURE 2: Heat maps of the genes at 1 (a), 3 (b), and 5 (c) days after SCI in the old vs. young groups. Horizontal axis represents each sample, and the vertical axis represents each gene. Blue and red colors represent low and high expression values, respectively.

precise mechanisms that delay fracture healing in the elderly remain unclear [25]. In the present study, bioinformatics analysis was used to determine molecular events and pathological states that occurred in the early phases of fracture healing at different organismal ages. GSE17825 database transcriptome data were collected from fracture calluses of old and young C57BL6 mice (8-month-old and 6-week-old mice) at 1,3 , and 5 days after fracture. Analysis of gene expression at these three time-points revealed DEGs between the subject groups, and KEGG pathway and GO enrichment analyses were subsequently performed. A PPI network was then constructed to further analyze molecular processes underlying fracture healing. The findings of these analyses may contribute to a better understanding of the effects of aging on fracture healing.

Because inflammation is a critical step in fracture healing, any disruption of this process can negatively affect overall healing [9]. Our GO enrichment analyses revealed that DEGs expressed at day 3 were most enriched in antigen processing and peptide or polysaccharide antigen presentation via MHC class II. Moreover, KEGG pathway analyses showed that upregulated DEGs were primarily associated with inflammatory response-related processes (CAMs, rheumatoid arthritis, and leukocyte transendothelial migration) at 5 days after fracture. These results are consistent with those of previous studies where aging was shown to affect fracture healing via a chronic increase in proinflammatory status; the notion of "inflamm-aging" has been used to describe this status in the elderly [26]. This elevated inflammatory status is associated with poorer fracture healing outcomes [27-29]; however, the mechanism remains unknown. Greater knowledge of mechanisms of this inflammatory process can be applied to positively impact fracture healing.

MSC differentiation is another key process in fracture healing, and understanding the mechanisms leading to delayed cell differentiation is important for understanding the cause of reduced skeletal regeneration with age [30,31]. Here KEGG pathway, GO enrichment, and PPI network analyses demonstrated that the Wnt signaling pathway was downregulated at 3 days after fracture. The Wnt signaling pathway plays major roles in both skeletal development and homeostasis [32-34]. In early pluripotent MSCs, Wnt $/ \beta$ catenin signaling must be precisely regulated to facilitate the differentiation of osteoblasts; dysregulation of this pathway alters the normal bone healing response $[35,36]$. In aged animals, a reduction in MSC osteoblast differentiation ability or preferential differentiation into adipocytes may be attributable to the downregulation of the Wnt signaling pathway. Therefore, pharmacologic agents that target this pathway can yield effective therapies to improve bone repair in the elderly.

KEGG pathway enrichment and PPI network analysis revealed that the downregulated DEGs were primarily related to vascularization-associated functions (platelet activation, hematopoietic cell lineage, and VEGF signaling pathway) at 5 days after fracture. Vascularization is a complex process that is essential for successful bone repair [7]. Decreased vascular system function is likely to decrease the amount of oxygen 


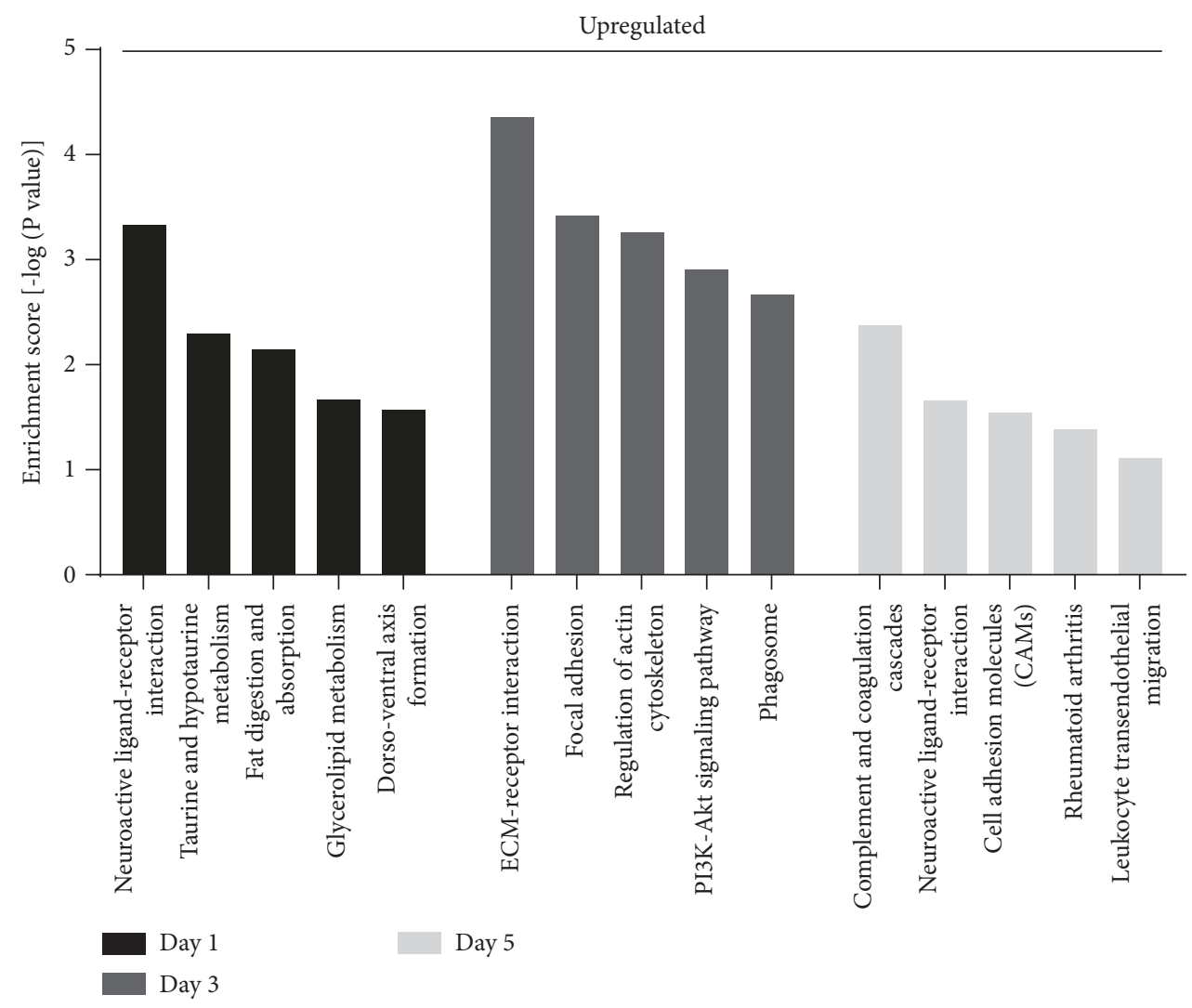

(a)

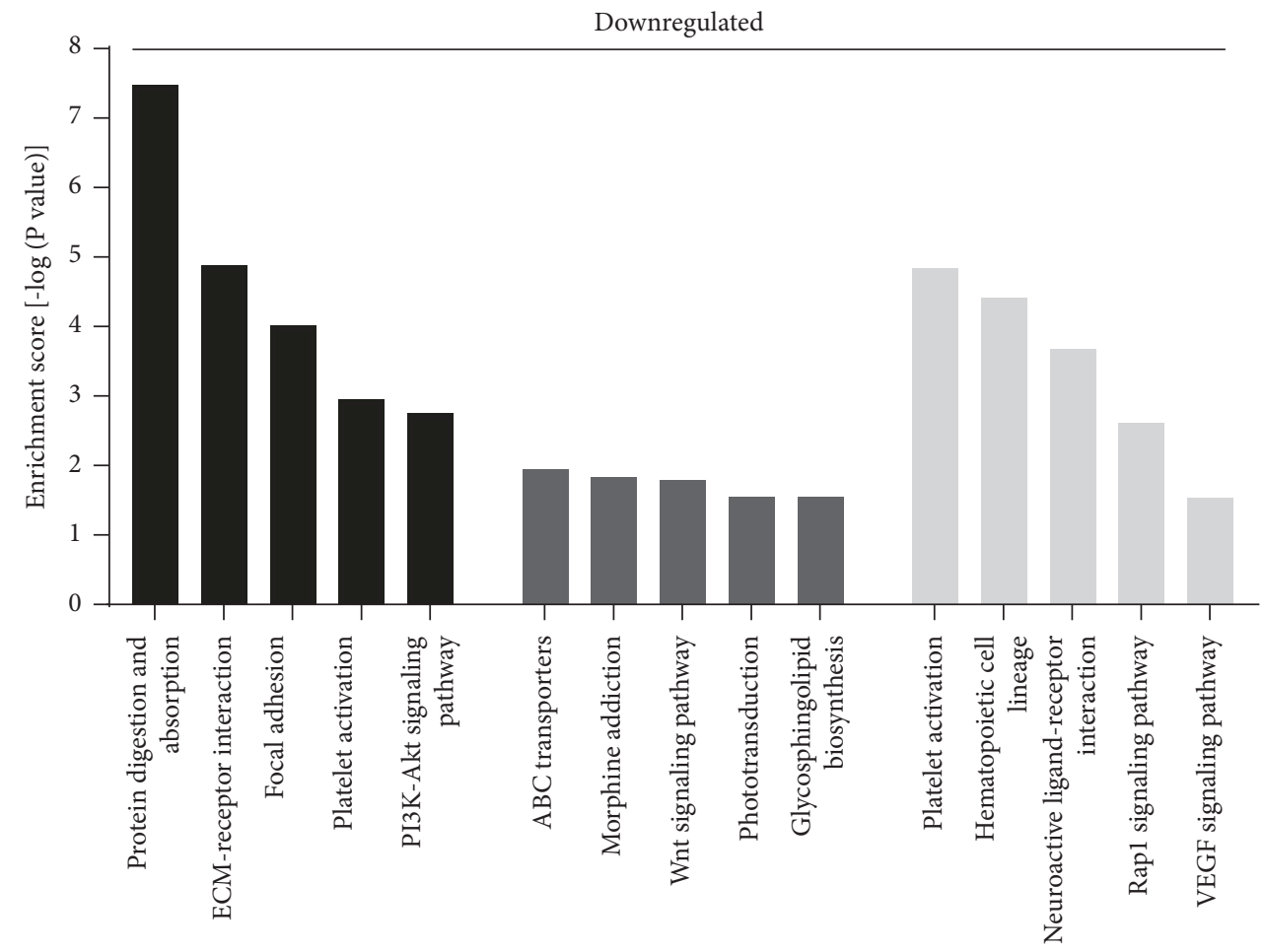

Day 1

Day 5

Day 3

(b)

FIGURE 3: Kyoto Encyclopedia of Genes and Genomes pathways of (a) upregulated and (b) downregulated DEGs at three time-points $(1,3$, and 5 days) following bone fracture. 
TABLE 1: GO terms enriched by DEGs at three time-points following bone fracture.

\begin{tabular}{|c|c|c|c|}
\hline Category & Term & Description & P-value \\
\hline \multicolumn{4}{|c|}{ Old vs young (day 1 ) } \\
\hline & GO:0030199 & collagen fibril organization & 8.90E-09 \\
\hline & GO:0001501 & skeletal system development & $3.32 \mathrm{E}-06$ \\
\hline & GO:0009888 & tissue development & $5.61 \mathrm{E}-06$ \\
\hline & GO:0051241 & negative regulation of multicellular organismal process & $6.96 \mathrm{E}-06$ \\
\hline & GO:0010721 & negative regulation of cell development & $2.37 \mathrm{E}-05$ \\
\hline & GO:0009887 & animal organ morphogenesis & 2.37E-05 \\
\hline & GO:0060343 & trabecula formation & $1.04 \mathrm{E}-04$ \\
\hline & GO:0060346 & bone trabecula formation & $1.08 \mathrm{E}-04$ \\
\hline & GO:0071229 & cellular response to acid chemical & $1.36 \mathrm{E}-04$ \\
\hline & GO:0001101 & response to acid chemical & 1.61E-04 \\
\hline \multicolumn{4}{|c|}{ Old vs young (day 3 ) } \\
\hline & GO:0002504 & antigen processing and presentation of peptide or polysaccharide antigen via $\mathrm{MHC}$ class II & $5.58 \mathrm{E}-07$ \\
\hline & GO:0071805 & potassium ion transmembrane transport & 7.41E-07 \\
\hline & GO:0071804 & cellular potassium ion transport & 7.41E-07 \\
\hline & GO:0055085 & transmembrane transport & $1.83 \mathrm{E}-05$ \\
\hline & GO:0015672 & monovalent inorganic cation transport & 1.29E-04 \\
\hline & GO:1990118 & sodium ion import into cell & 1.77E-04 \\
\hline & GO:0010876 & lipid localization & 1.91E-04 \\
\hline & GO:2000054 & negative regulation of Wnt signaling pathway involved in dorsal/ventral axis & $1.94 \mathrm{E}-04$ \\
\hline & GO:0034113 & heterotypic cell-cell adhesion & $2.40 \mathrm{E}-04$ \\
\hline & GO:0034220 & ion transmembrane transport & $2.59 \mathrm{E}-04$ \\
\hline \multicolumn{4}{|c|}{ Old vs young (day 5 ) } \\
\hline & GO:0010951 & negative regulation of endopeptidase activity & 1.61E-08 \\
\hline & GO:0098916 & anterograde trans-synaptic signaling & $5.80 \mathrm{E}-07$ \\
\hline & GO:0099537 & trans-synaptic signaling & $5.98 \mathrm{E}-07$ \\
\hline & GO:0099536 & synaptic signaling & 7.67E-07 \\
\hline & GO:0006950 & response to stress & 1.15E-06 \\
\hline & GO:0051346 & negative regulation of hydrolase activity & $2.41 \mathrm{E}-06$ \\
\hline & GO:0007270 & neuron-neuron synaptic transmission & $1.00 \mathrm{E}-05$ \\
\hline & GO:0006955 & immune response & 1.81E-05 \\
\hline & GO:0006508 & proteolysis & $2.27 \mathrm{E}-05$ \\
\hline & GO:0007268 & chemical synaptic transmission & $4.16 \mathrm{E}-05$ \\
\hline
\end{tabular}

GO, gene ontology; DEGs, differentially expressed genes.

present at the fracture site, impair nutrient exchange, and potentially cause problems during the recruitment of cells to the fracture site $[7,16]$. These molecular changes may contribute to delayed fracture healing in the elderly relative to that in juveniles.

Notably, DEGs at day 5 after fracture were primarily enriched in synaptic-related functions. The exact role of sensory nerves in fracture healing remains elusive [37-39] because few studies have focused on age-related changes within the central nervous system or sensory nerves in bone repair regulation. Such synaptic alterations warrant further investigation.

One limitation of the present study is that the analyzed raw dataset did not include transcriptome data from 2, 3 , 4 , or 5 weeks following fracture, limiting conclusions about changes in molecular processes after bone fracture over time. Taken together, our findings demonstrate that elevated inflammatory status, a dysregulated Wnt signaling pathway, and the downregulation of vascularization-associated functions and synaptic-related processes play essential roles in fracture healing in the elderly. Further studies are required to determine the mechanisms by which these molecular events affect fracture healing.

\section{Data Availability}

The original data used to support the findings of this study are available at GEO dataset (GSE17825).

\section{Conflicts of Interest}

The authors declare that they have no conflicts of interest. 


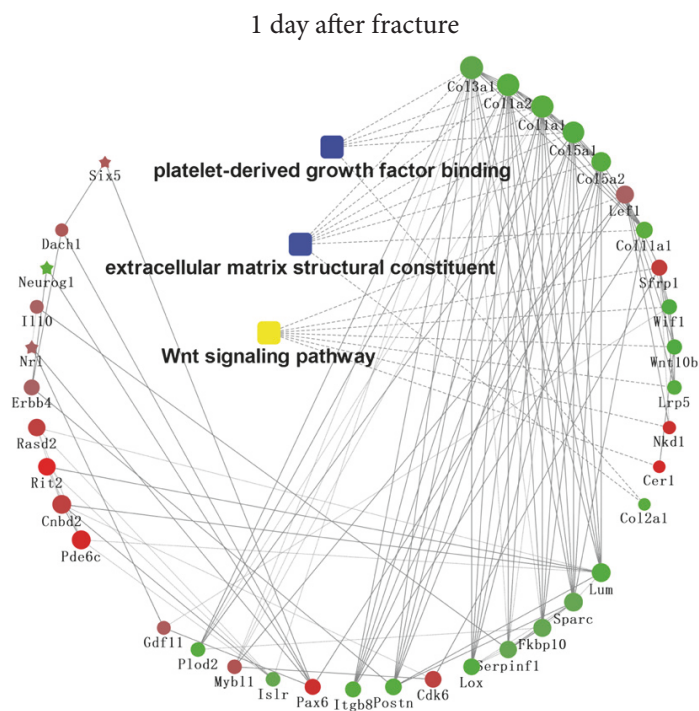

Low low
Fold change

GO/KEGG Terms

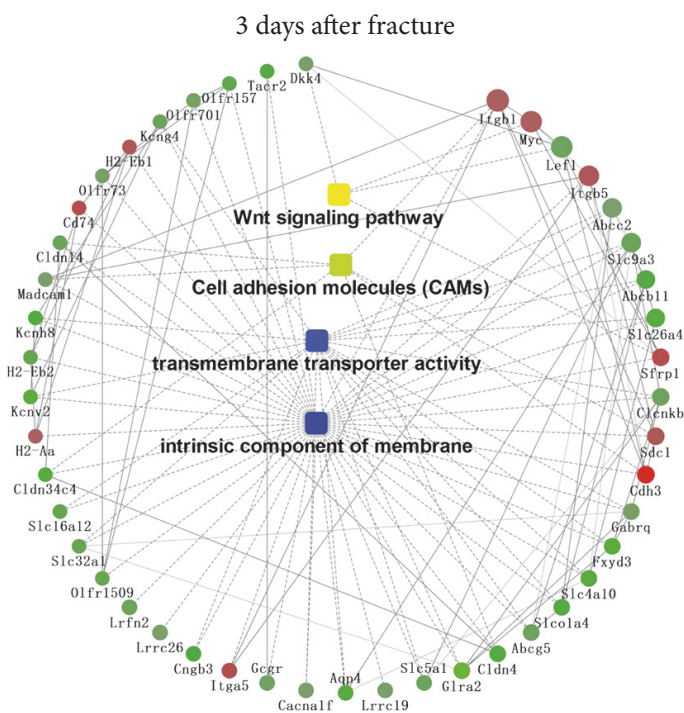

Low low high High

Fold change

GO/KEGG Terms

(a)

(b)

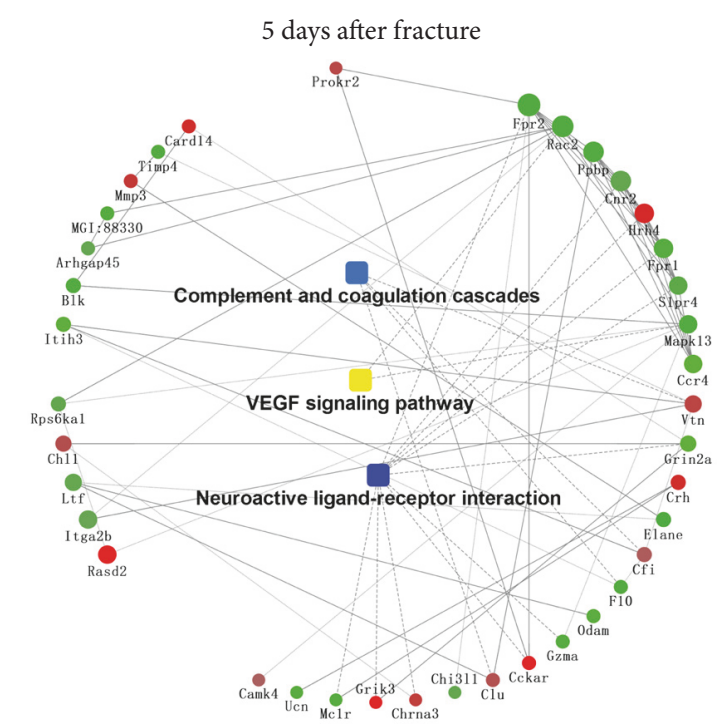

Low low hold change
Fold

GO/KEGG Terms

(c)

FIgURE 4: PPI networks based on DEGs. The gradient colors from green to red indicate increasing P values. Circular nodes indicate genes/proteins. Rectangular nodes indicate a biological process or a KEGG pathway. DEGs, differentially expressed genes; PPI, protein-protein interaction; KEGG, Kyoto Encyclopedia of Genes and Genomes. 


\section{Authors' Contributions}

Guo-Yong Yin and Ming-Xin Xue designed the present study. Shu-Jie Zhao, Fan-Qi Kong, Ying Chen, Shuai Zhou, and Jin Fan performed the data analysis and statistical analysis. ShuJie Zhao and Fan-Qi Kong wrote and revised the manuscript. Guo-Yong Yin supervised the present study. All authors read and approved the final manuscript. Shu-Jie Zhao and Fan-Qi Kong contributed equally to this work.

\section{Acknowledgments}

We would like to thank Dr Jian Chen (Department of Orthopedics, The First Affiliated Hospital of Nanjing Medical University) for assistance with the present study. The present study was supported by grants from the National Natural Science Foundation of China (grant nos. 8177235, 81520108018, and 81472080), the Jiangsu Committee of Science and Technology-Social Development Plan (grant no. BE2017755), Postgraduate Research \& Practice Innovation Program of Jiangsu Province (grant no. SJCX18_0430) and the Nanjing Committee of Science and Technology (grant no. 201505005).

\section{References}

[1] R. Iorio, W. J. Robb, W. L. Healy et al., "Orthopaedic surgeon workforce and volume assessment for total hip and knee replacement in the United States: Preparing for an epidemic," The Journal of Bone \& Joint Surgery, vol. 90, no. 7, pp. 1598-1605, 2008.

[2] UScensus, "U.S. Department of Commerce, Economics and Statistics Administration, U.S. Census Bureau, Washington. 2015," http://www.census.gov/content/dam/Census/library/publications/2015/demo/p25-1143.pdf.

[3] G. Jones, T. Nguyen, P. N. Sambrook, P. J. Kelly, C. Gilbert, and J. A. Eisman, "Symptomatic fracture incidence in elderly men and women: The Dubbo osteoporosis epidemiology study (DOES)," Osteoporosis International, vol. 4, no. 5, pp. 277-282, 1994.

[4] "Office of the Surgeon G: Reports of the Surgeon General," in Bone Health and Osteoporosis: A Report of the Surgeon General, Office of the Surgeon General (US), Rockville, MD, USA, 2004.

[5] E. Green, J. D. Lubahn, and J. Evans, "Risk factors, treatment, and outcomes associated with nonunion of the midshaft humerus fracture," Journal of Surgical Orthopaedic Advances, vol. 14, no. 2, pp. 64-72, 2005.

[6] C. Tzioupis and P. V. Giannoudis, "Prevalence of long-bone non-unions," Injury, vol. 38, no. 2, pp. S3-S9, 2007.

[7] L. Claes, S. Recknagel, and A. Ignatius, "Fracture healing under healthy and inflammatory conditions," Nature Reviews Rheumatology, vol. 8, no. 3, pp. 133-143, 2012.

[8] R. Marsell and T. A. Einhorn, "The biology of fracture healing," Injury, vol. 42, no. 6, pp. 551-555, 2011.

[9] E. Gibon, L. Lu, and S. B. Goodman, "Aging, inflammation, stem cells, and bone healing," Stem Cell Research \& Therapy, vol. 7, article 44, 2016.

[10] L. C. Gerstenfeld, T.-J. Cho, T. Kon et al., "Impaired intramembranous bone formation during bone repair in the absence of tumor necrosis factor-alpha signaling," Cells Tissues Organs, vol. 169, no. 3, pp. 285-294, 2001.
[11] P. Hernigou, A. Poignard, S. Zilber, and H. Rouard, "Cell therapy of hip osteonecrosis with autologous bone marrow grafting," Indian Journal of Orthopaedics, vol. 43, no. 1, pp. 4045, 2009.

[12] A. J. Stranks, A. L. Hansen, I. Panse et al., "Autophagy Controls Acquisition of Aging Features in Macrophages," Journal of Innate Immunity, vol. 7, no. 4, pp. 375-391, 2015.

[13] C. A. Cecílio, E. H. Costa, P. U. Simioni, D. L. Gabriel, and W. M. S. C. Tamashiro, "Aging alters the production of iNOS, arginase and cytokines in murine macrophages," Brazilian Journal of Medical and Biological Research, vol. 44, no. 7, pp. 671-681, 2011.

[14] A. Sanghani-Kerai, M. Coathup, S. Samazideh et al., "Osteoporosis and ageing affects the migration of stem cells and this is ameliorated by transfection with CXCR4," Bone \& Joint Research, vol. 6, no. 6, pp. 358-365, 2017.

[15] Z. Xing, C. Lu, D. Hu et al., "Multiple roles for CCR2 during fracture healing," Disease models mechanisms, vol. 3, pp. 451458, 2010.

[16] C. Lu, E. Hansen, A. Sapozhnikova, D. Hu, T. Miclau, and R. S. Marcucio, "Effect of age on vascularization during fracture repair," Journal of Orthopaedic Research, vol. 26, no. 10, pp. 13841389, 2008.

[17] T. Shimada, Y. Takeshita, T. Murohara et al., "Angiogenesis and vasculogenesis are impaired in the precocious-aging klotho mouse," Circulation, vol. 110, no. 9, pp. 1148-1155, 2004.

[18] H. Ito, "Chemokines in mesenchymal stem cell therapy for bone repair: A novel concept of recruiting mesenchymal stem cells and the possible cell sources," Modern Rheumatology, vol. 21, no. 2, pp. 113-121, 2011.

[19] A. Phillips, "Overview of the fracture healing cascade," Injury, vol. 36, no. 3, pp. S5-S7, 2005.

[20] A. Schindeler, M. M. McDonald, P. Bokko, and D. G. Little, "Bone remodeling during fracture repair: the cellular picture," Seminars in Cell \& Developmental Biology, vol. 19, no. 5, pp. 459466, 2008.

[21] B. S. Carvalho and R. A. Irizarry, "A framework for oligonucleotide microarray preprocessing," Bioinformatics, vol. 26, no. 19, Article ID btq431, pp. 2363-2367, 2010.

[22] D. W. Huang, B. T. Sherman, and R. A. Lempicki, "Systematic and integrative analysis of large gene lists using DAVID bioinformatics resources," Nature Protocols, vol. 4, no. 1, pp. 44-57, 2009.

[23] P. Shannon, A. Markiel, O. Ozier et al., "Cytoscape: a software Environment for integrated models of biomolecular interaction networks," Genome Research, vol. 13, no. 11, pp. 2498-2504, 2003.

[24] S. Zhao, W. Zhou, J. Chen, Y. Luo, and G. Yin, "Bioinformatics analysis of the molecular mechanisms underlying traumatic spinal cord injury," Molecular Medicine Reports, 2018.

[25] C. Lu, T. Miclau, D. Hu et al., "Cellular basis for age-related changes in fracture repair," Journal of Orthopaedic Research, vol. 23, no. 6, pp. 1300-1307, 2005.

[26] S. Xia, X. Zhang, S. Zheng et al., "An Update on InflammAging: Mechanisms, Prevention, and Treatment," Journal of Immunology Research, vol. 2016, Article ID 8426874, 12 pages, 2016.

[27] K. Schmidt-Bleek, H. Schell, N. Schulz et al., "Inflammatory phase of bone healing initiates the regenerative healing cascade," Cell and Tissue Research, vol. 347, no. 3, pp. 567-573, 2012.

[28] M. V. Thomas and D. A. Puleo, "Infection, inflammation, and bone regeneration: a paradoxical relationship," Journal of Dental Research, vol. 90, no. 9, pp. 1052-1061, 2011. 
[29] M. I. Dishowitz, P. L. Mutyaba, J. D. Takacs et al., "Systemic inhibition of canonical notch signaling results in sustained callus inflammation and alters multiple phases of fracture healing," PLoS ONE, vol. 8, no. 7, 2013.

[30] S. Sethe, A. Scutt, and A. Stolzing, "Aging of mesenchymal stem cells," Ageing Research Reviews, vol. 5, no. 1, pp. 91-116, 2006.

[31] D. Duscher, R. C. Rennert, M. Januszyk et al., "Aging disrupts cell subpopulation dynamics and diminishes the function of mesenchymal stem cells," Scientific Reports, vol. 4, article 7144, 2014.

[32] J. B. Regard, Z. Zhong, B. O. Williams, and Y. Yang, "Wnt signaling in bone development and disease: making stronger bone with Wnts," Cold Spring Harbor Perspectives in Biology, vol. 4, no. 12, 2012.

[33] Z. Zhong, N. J. Ethen, and B. O. Williams, "WNT signaling in bone development and homeostasis," Wiley Interdisciplinary Reviews: Developmental Biology, vol. 3, no. 6, pp. 489-500, 2014.

[34] M. A. Rudnicki and B. O. Williams, "Wnt signaling in bone and muscle," Bone, vol. 80, pp. 60-66, 2015.

[35] H. Xu, J. Duan, D. Ning et al., "Role of Wnt signaling in fracture healing," BMB Reports, vol. 47, no. 12, pp. 666-672, 2014.

[36] D. Silkstone, H. Hong, and B. A. Alman, " $\beta$-catenin in the race to fracture repair: in it to Wnt," Nature Clinical Practice Rheumatology, vol. 4, no. 8, pp. 413-419, 2008.

[37] Y. Zhang, J. Xu, and YC. Ruan, Implant-Derived Magnesium Induces Local Neuronal Production of CGRP to Improve BoneFracture Healing in Rats, vol. 22, 2016.

[38] D. Cadosch, O. P. Gautschi, M. Thyer et al., "Humoral factors enhance fracture-healing and callus formation in patients with traumatic brain injury," The Journal of Bone and Joint Surgery-American Volume, vol. 91, no. 2, pp. 282-288, 2009.

[39] M. Boes, M. Kain, S. Kakar et al., "Osteogenic effects of traumatic brain injury on experimental fracture-healing," The Journal of Bone and Joint Surgery-American Volume, vol. 88, no. 4, pp. 738-743, 2006. 


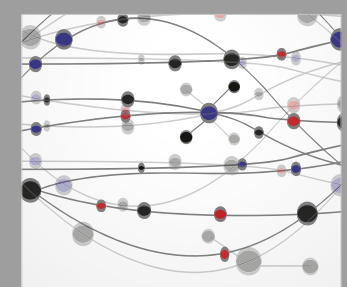

The Scientific World Journal
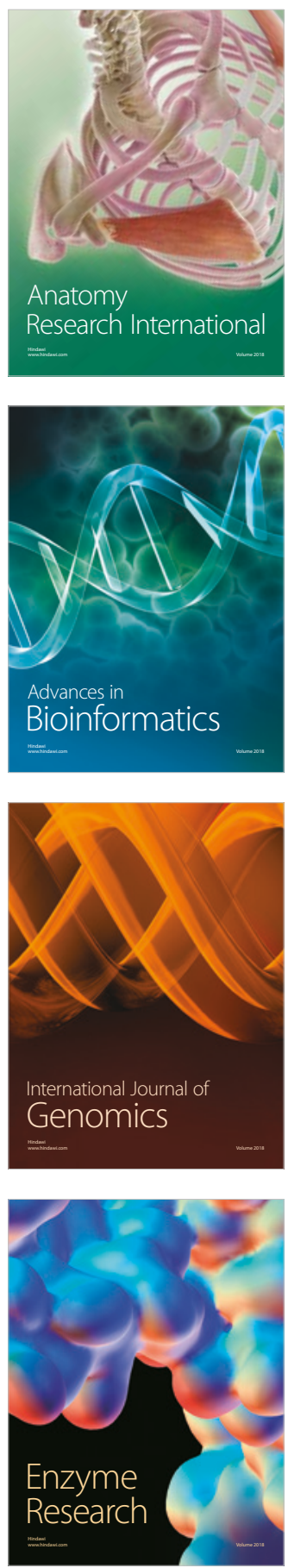
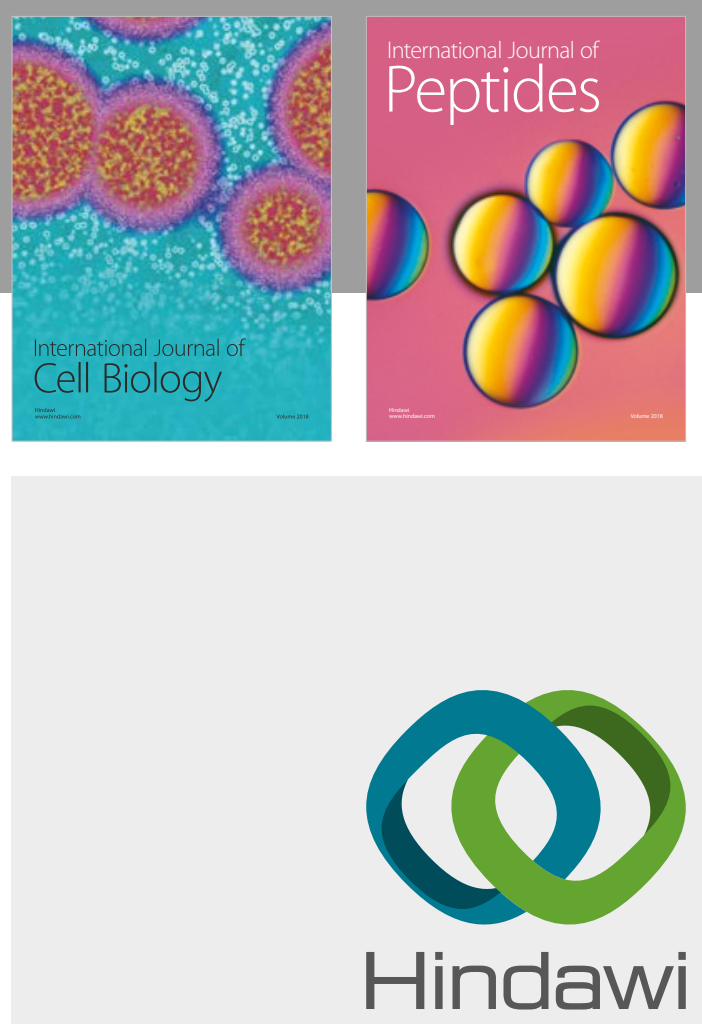

Submit your manuscripts at

www.hindawi.com
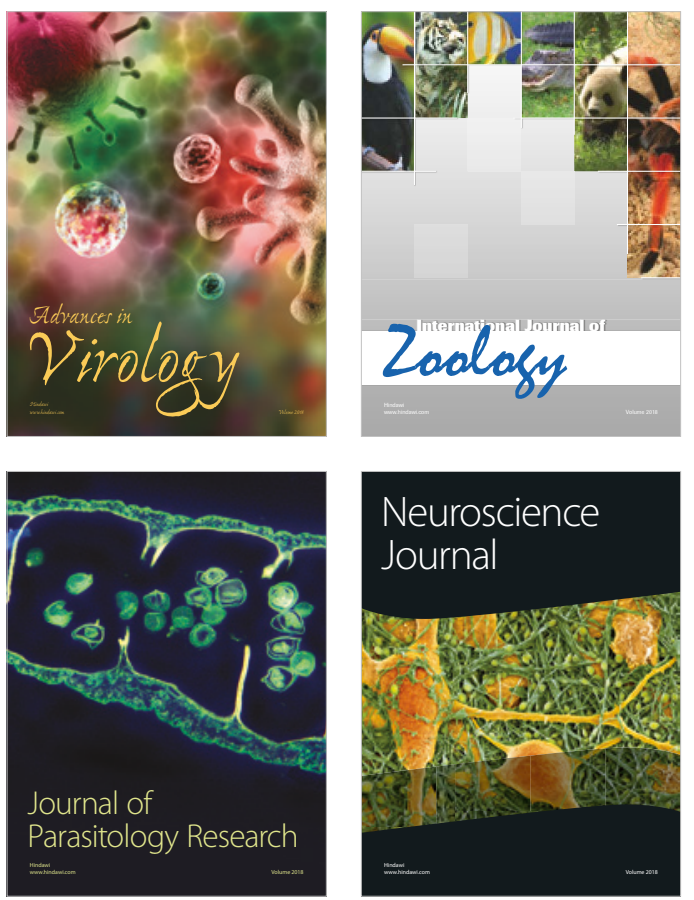
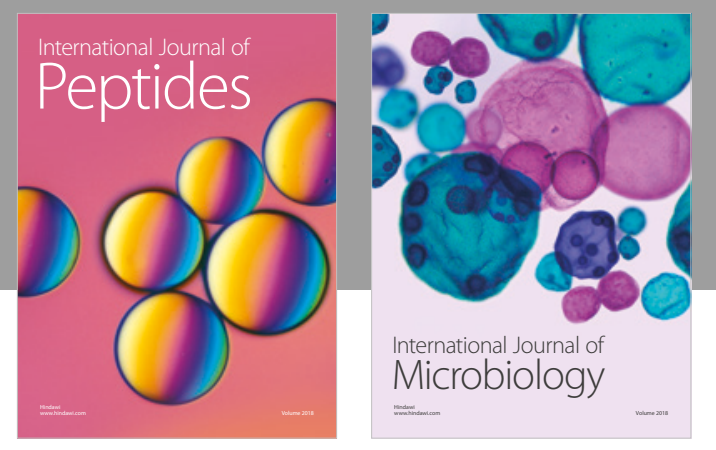

nternational Journal of Microbiology
Journal of
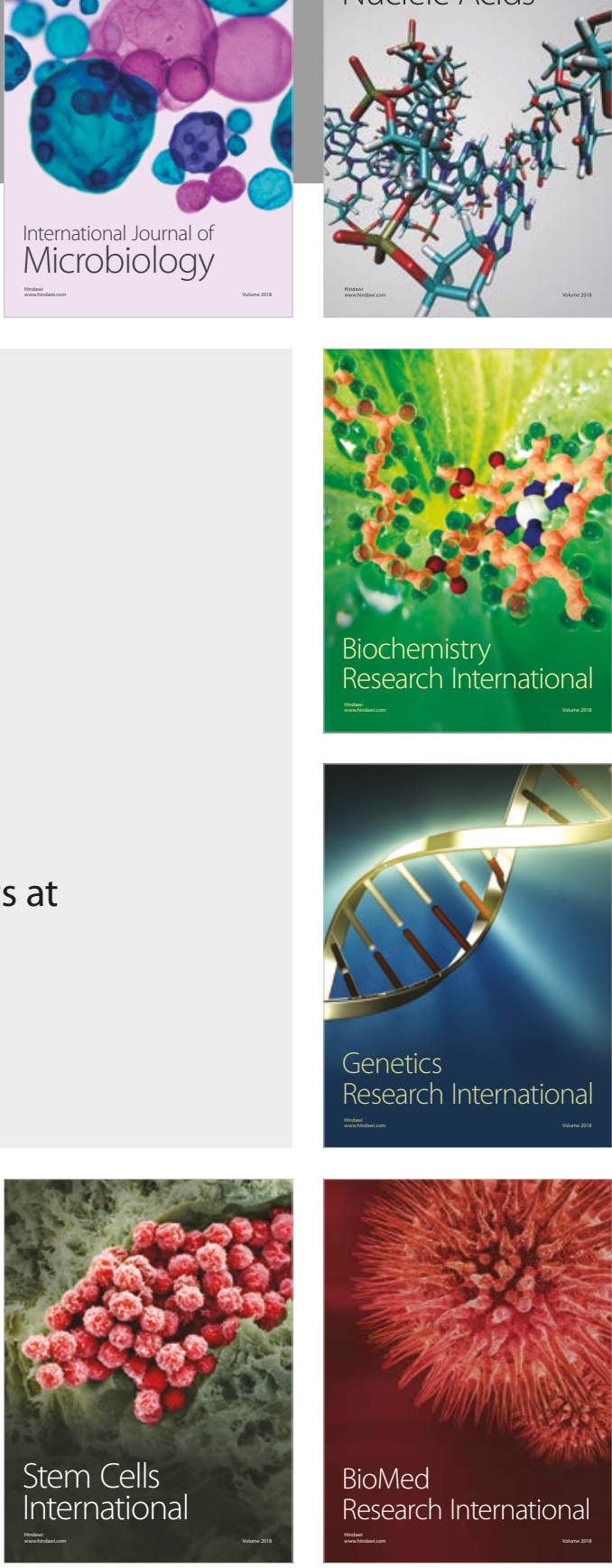
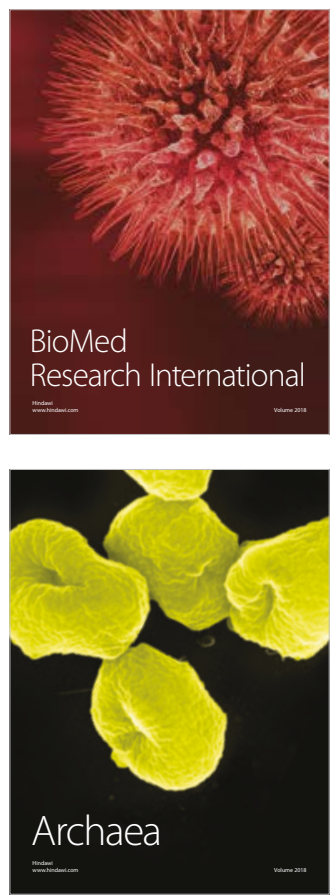